\title{
Prevalence of Cytomegalovirus infections in ulcerative rettocolitis patients, using PCR in whole blood and biopsies
}

\author{
Anna Todisco', Raffaele Melina², Pia Carmen Melillo', Maria Landi', Elio De Nisco', \\ Raffaele Ariola', Franca Romeo', Generoso Violano', Gaetano laquinto ${ }^{2}$ \\ I Unità Operativa Complessa di Virologia, Dip. Medicina di Laboratorio, AORN “S.G. Moscati”, Avellino \\ 2 Unità Operativa Complessa di Gastroenterologia, Dip. Chirurgia Generale e Specialistica, AORN “S.G. Moscati”, Avellino
}

Key words: CMV in RCU patients

Prevalenza di infezione da Cytomegalovirus in un gruppo di pazienti con rettocolite ulcerosa, valutata mediante PCR su sangue intero e biopsie

\section{SUMMARY}

Background. The relationship between CMV and inflammatory bowel disease (IBD) is not yet fully understood; the viral reactivation could be related to the prolonged steroid treatment of patients with ulcerative colitis (UC).

Study Design. Our study was conducted on 25 patients with reactivation of UC, all were screened for CMV research. CMV DNA was carried out by automatic extraction in whole blood and intestinal biopsies and amplified by RealTime PCR.

Objectives. We assessed the prevalence of CMV infection and clinical outcome, particularly in CMV positive patients treated with antiviral therapy.

Results. CMV DNA was detected in II/25 patients (44\%), 7 positive in both blood and biopsies, 2 positive biopsies, 2 positive in blood, but low charge.

I4 CMV DNA negative patients: 8 had remission with conventional therapy, 6 treated with IFX (of which 2 underwent colectomy).

I I CMV DNA positive patients: 7 had remission with conventional therapy, 3 treated with IFX+Ganciclovir, I with Ganciclovir, obtaining negative viral load and clinical remission.

Conclusion. The literature data available on the association CMV - IBD are partly conflicting.

The decision on treatment with antiviral therapy of CMV positive patients is based on course of disease. On the other hand, some patients had remission without antiviral therapy.

We considered viral load and resistance to conventional therapy to admit patients into antiviral treatment, getting acute phase remission.

There are many unanswered questions regarding management of CMV reactivation in UC patients, which need long-term follow-up studies and larger population.

\section{INTRODUZIONE}

Il legame esistente tra il Cytomegalovirus (CMV) e le malattie infiammatorie croniche intestinali (MICI) è probabilmente stretto ma non ancora del tutto chiarito. Il virus è stato descritto da numerosi autori come cofattore nella patogenesi delle MICI $(3,6,11)$. La prima descrizione di un'associazione tra malattia infiammatoria e CMV risale al 1961; negli anni successivi in letteratura (13, $14,16)$ vi è stato un susseguirsi di segnalazioni riguardanti il riscontro del virus nelle MICI, attribuendone un coinvolgimento sia in termini di esordio, sia di riattivazione che di severità di decorso.

L'immunodepressione indotta dalla stessa malattia infiammatoria o dai farmaci utilizzati per il mantenimento della remissione può influire sulla riattivazione del virus (1).

Il virus riconosce come sede di replicazione ideale, oltre che le cellule endoteliali, quelle della linea monocito-macrofagica, i leucociti, anche le cellule rapidamente proliferanti come quelle contenute nel tessuto di granulazione, in zone sede di infiammazione o nel fondo di ulcere, che sono un segno distintivo delle rettocoliti ulcerose. Si innescherebbe così un processo autoalimentato (8): le proteine virali hanno la capacità di attivare citochine pro-infiammatorie, come INF $\gamma$ e TNF $\alpha$, responsabili del mantenimento di un'infiammazione locale e dell'attivazione immune (Figura I); il TNF $\alpha$ possiede, a sua volta, come risulta da studi sperimentali, la capacità di incrementare la 
produzione di CMV attraverso l'attivazione del "major immediate early promoter", che regola la cascata trascrizionale dei geni necessari alla replicazione virale $(2,15)$.

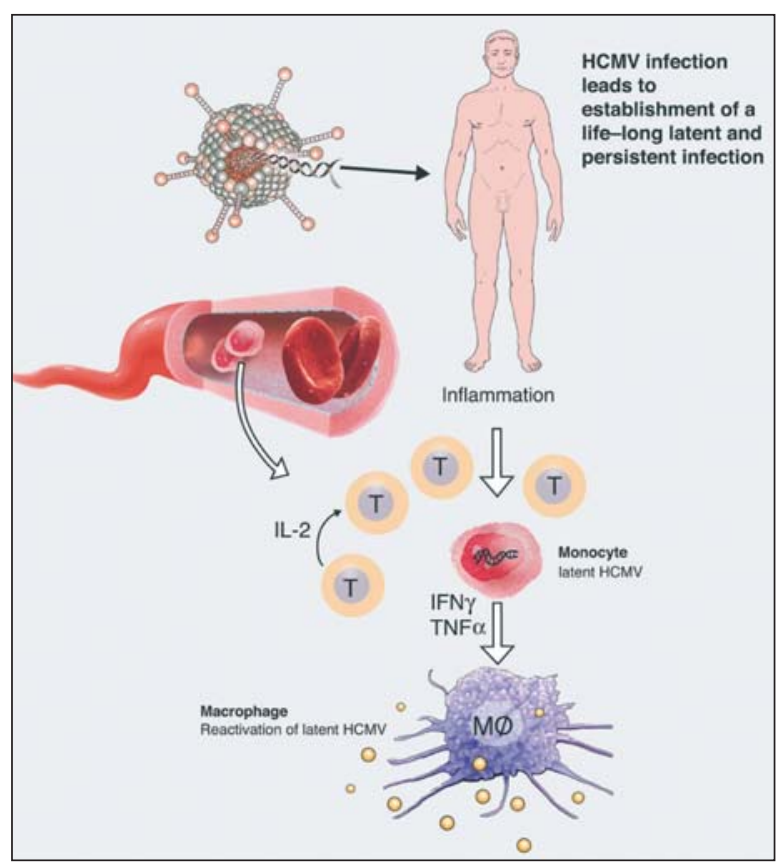

Figura I. A primary human cytomegalovirus (HCMV) infection leads to establishment of a life-long latent and persistent infectio (da Blackwell Publishing Ltd Journal of Internal Medicine 259: 219-246, 2006).
Ci sono studi che suggeriscono che la presenza di CMV nelle RCU sia dovuta al tropismo del virus verso le aree di displasia e infiammazione, ma che non ci sia un'implicazione reale nel processo patologico.

I sostenitori dell'ipotesi "Innocent Bystander" (10) affermano che la presenza di CMV non contribuisce all'aumento della morbilità e mortalità della malattia di base, e che la terapia antivirale può eliminare il virus senza alterare il decorso della sottostante malattia attiva $(5,12)$.

Altri autori, invece, asseriscono che l'infezione deve essere trattata con terapia antivirale (Ganciclovir o Foscarnet), al fine di ridurre la morbilità e la mortalità delle RCU $(4,7)$ (Figura II).

È stata formulata poi un'ipotesi intermedia tra le due descritte, secondo cui in caso di bassa carica virale l'infezione da CMV non può significativamente contribuire ai sintomi della colite attiva, tuttavia, un'elevata carica potrebbe evolvere verso un'ischemia microvascolare, esagerata risposta infiammatoria e alterata emostasi, aggravando le aree infiammate già vulnerabili e conducendo al peggioramento della sottostante RCU, all'aumento della morbilità e della mortalità (9).

Gli obiettivi del nostro lavoro sono stati la valutazione della prevalenza dell'infezione (mediante metodica PCR su sangue e biopsie intestinali) in pazienti con RCU riacutizzata e dell'outcome clinico, in particolare nei pazienti CMV positivi trattati con terapia antivirale.

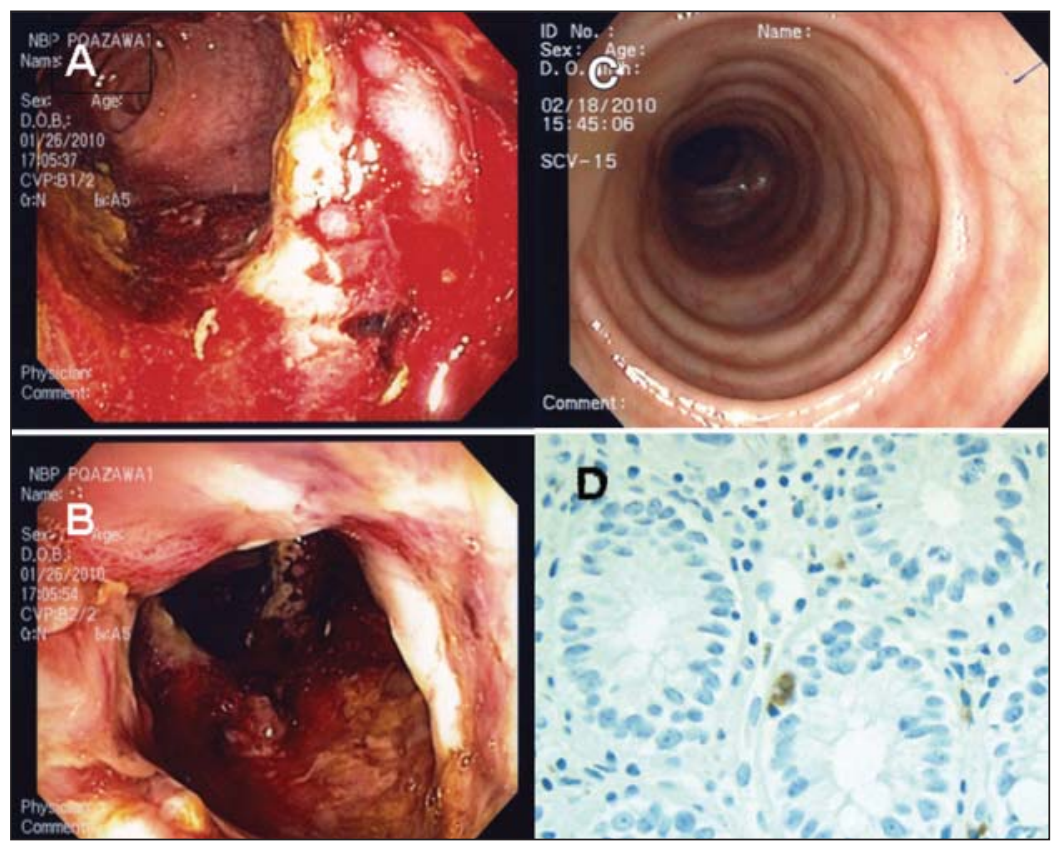

Figura II. Sigmoidoscopy and cytomegalovirus immunostaining. Photos taken during sigmoidoscopy before (A, B) and after (C) treatment with intravenous ganciclovir. Immunostaining performed on the biopsy taken during colonoscopy shown in panel $A$ stained positive for cytomegalovirus (D), original magnification $\times 100$ (da Can J Infect Dis Med Microbiol Vol 22, No 3 - 20 I I; Rajal Khan et al., Cytomegalovirus colitis following azacitidine therapy).

\section{METODI}

Il nostro studio è stato condotto su 25 pazienti consecutivi con RCU, ricoverati dal 2010 al 2012 presso l'Unità Operativa di Gastroenterologia dell'AORN "S.G. Moscati" di Avellino, per riattivazione severa della colite. Tutti sono stati sottoposti a screening per ricerca di CMV al momento del ricovero.

La ricerca del CMV DNA è stata effettuata mediante estrazione automatica di DNA su strumento Qiacube, utilizzando QIAamp DNA BLOOD MiniKit (Qiagen) per il sangue intero e QIAamp $D N A$ MiniKit (Qiagen) per le biopsie. Il DNA estratto è stato amplificato mediante RealTime PCR utilizzando $Q-C M V$ RealTime Complete Kit (Nanogen), basato sulla tecnologia Taqman, su strumento Applied Biosystem 7300. 


\section{RISULTATI}

Il CMV DNA è stato riscontrato in 11 dei 25 pazienti con RCU riacutizzata (44\%) (Tabella 1); 7 sono risultati positivi sia su sangue periferico che sulle biopsie rettali, 2 positivi solo sulle biopsie, 2 solo su sangue ma a bassa carica. Tutti i pazienti erano in trattamento con farmaci steroidei all'ingresso. Dei 14 pazienti CMV negativi, 8 sono andati in remissione con terapia convenzionale, 6 sono stati trattati con IFX di cui 2 sottoposti a colectomia per mancata risposta alla terapia e rapido peggioramento clinico. Degli 11 pazienti CMV DNA positivi, 7 sono andati in remissione con terapia convenzionale, 3 sono stati trattati con IFX + terapia antivirale (Ganciclovir), 1 solo con terapia antivirale, ottenendo negativizzazione della viral load e remissione clinica. Il criterio adoperato per la scelta del trattamento con terapia antivirale è stato il riscontro di CMV positività su biopsia e su sangue intero con viral load misurabile $(>1111 \mathrm{copie} / \mathrm{ml})$, in pazienti resistenti alla terapia convenzionale.

\section{DISCUSSIONE}

È noto che il CMV svolge un ruolo patogenetico importante nelle condizioni di immunodeficienza; negli ultimi anni il virus ha suscitato molto interesse anche per il suo coinvolgimento nelle patologie infiammatorie croniche.

I dati di letteratura disponibili sull'associazione tra il virus e le malattie infiammatorie intestinali e i suoi meccanismi sono in parte discordanti.

L'aspetto che ancora non è chiaro è se la presenza del virus nelle biopsie intestinali di pazienti con colite attiva svolga effettivamente un ruolo di cofattore nel mantenimento dell'infiammazione stessa. Quello che si sa è che il virus nel momento in cui interagisce con l'ospite, scatena un'attivazione di citochine ad azione pro-infiammatoria che probabilmente mantengono o talvolta innescano un'infiammazione persistente.

La decisione sul trattamento con terapia antivirale dei pazienti RCU CMV positivi resta discrezionale in base all'andamento della malattia. D'altro canto in alcuni studi effettuati in tali pazienti la remissione è stata ottenuta anche senza terapia antivirale.

Il criterio da noi adottato, che ha tenuto conto della carica virale riscontrata e della resistenza alla terapia convenzionale, ha trovato riscontro

Tabella I. Tabella riepilogativa CMV DNA da sangue e biopsie su pazienti con RCU riacutizzata.

\begin{tabular}{|c|c|c|c|c|c|}
\hline & \multicolumn{2}{|c|}{ VIROLOGIA } & \multirow{2}{*}{$\begin{array}{c}\text { TERAPIA } \\
\text { FARMACOLOGIC } \\
\text { A }\end{array}$} & \multicolumn{2}{|l|}{ ESITO } \\
\hline PAZIENTE & CMV S.I. & $\begin{array}{c}\text { CMV } \\
\text { BIOPSIA }\end{array}$ & & $\begin{array}{l}\text { REMISSIONE della } \\
\text { RIACUTIZZAZIONE }\end{array}$ & CHIRURGIA \\
\hline I. & NON RIL & NEG & Conv & $\mathrm{SI}$ & \\
\hline 2. & NON RIL & & Conv. & $\mathrm{SI}$ & \\
\hline 3. & NON RIL & & Conv & $\mathrm{SI}$ & \\
\hline 4. & $<556$ & NEG & Conv & $\mathrm{SI}$ & \\
\hline 5. & & NEG & Conv & $\mathrm{SI}$ & \\
\hline 6. & NON RIL & NEG & Conv + IFX & SI & \\
\hline 7. & NON RIL & NEG & Conv + IFX & $\mathrm{SI}$ & \\
\hline 8. & NON RIL & POS & Conv & $\mathrm{SI}$ & \\
\hline 9. & $\begin{array}{c}2778 \\
1^{\circ} \text { controllo: }<556 \\
2^{\circ} \text { controllo: NON RIL }\end{array}$ & POS & $\begin{array}{l}\text { Conv }+ \text { antivirali } \\
\text { (Ganciclovir) }\end{array}$ & SI & \\
\hline 10. & NON RIL & & Conv & $\mathrm{SI}$ & \\
\hline 11. & $\overline{\text { NON RIL }}$ & POS & Conv & SI & \\
\hline 12. & NON RIL & & Conv + IFX & $\mathrm{SI}$ & \\
\hline 13. & 1155 & POS & Conv & $\mathrm{SI}$ & \\
\hline 14. & $\begin{array}{c}1177 \\
1{ }^{\circ} \text { controllo: NON RIL }\end{array}$ & POS & $\begin{array}{l}\text { Conv + antivirale } \\
\text { (Ganciclovir), IFX }\end{array}$ & $\mathrm{SI}$ & \\
\hline 15. & 33780 & POSITIVA & Conv & $\mathrm{SI}$ & \\
\hline 16. & $\begin{array}{c}11112 \\
1^{\circ} \text { controllo: NON RIL } \\
2^{\circ} \text { controllo: NON RIL }\end{array}$ & $\begin{array}{l}\text { POS } \\
\text { NEG }\end{array}$ & $\begin{array}{l}\text { Conv + antivirale } \\
\text { (Ganciclovir), IFX }\end{array}$ & $\mathrm{SI}$ & \\
\hline 17. & $\begin{array}{c}11112 \\
1{ }^{\circ} \text { controllo: NON RIL }\end{array}$ & $\begin{array}{l}\text { POS } \\
\text { NEG }\end{array}$ & $\begin{array}{l}\text { Conv + antivirale } \\
\text { (Ganciclovir), IFX }\end{array}$ & SI & \\
\hline 18. & NON RIL & & Conv & $\mathrm{SI}$ & \\
\hline 19. & $<556$ & NEG & Conv & $\mathrm{SI}$ & \\
\hline 20. & NON RIL & NEG & Conv & SI & \\
\hline 21. & NON RIL & & Conv + IFX & $\mathrm{SI}$ & \\
\hline 22. & NON RIL & NEG. & Conv + IFX & & $\mathrm{SI}$ \\
\hline 23. & $<556$ & POS & Conv & $\mathrm{SI}$ & \\
\hline 24. & NON RIL & NEG & Conv + IFX & & $\mathrm{SI}$ \\
\hline 25. & NON RIL & & Conv & $\mathrm{SI}$ & \\
\hline
\end{tabular}


clinico nella remissione della fase acuta della malattia dopo il trattamento antivirale.

Rimangono molte questioni irrisolte per quanto riguarda la gestione della riattivazione del CMV nei pazienti affetti da RCU, che devono essere affrontate con studi di follow up a lungo termine e su una popolazione più ampia. Ulteriori indagini sul comportamento e sulla patogenicità del virus sono necessarie per comprendere quando e se trattare l'infezione, e stabilire così una comune linea guida da adottare in corso di riattivazione di colite CMV associata.

\section{BIBLIOGRAFIA}

1. Epple HJ. Therapy- and non-therapy-dependent infectious complications in inflammatory bowel disease. Dig Dis 2009; 27(4): 555-9. Epub 2009 Nov 4.

2. Geist LJ, Hopkins HA, Dai LY, He B, Monick MM, Hunninghake GW. Cytomegalovirus modulates transcription factors necessary for the activation of the tumor necrosis factor-alpha promoter. Am J Respir Cell Mol Biol. 1997 Jan; 16: 31-7.

3. Gilat T, Hacohen P, Lilos P. Childhood factors in ulcerative colitis and Crohn's disease. Scand $J$ Gastroenterol 1987; 22: 1009-24.

4. Hamlin PJ, Shah MN, Scott N, Wyatt JI, Howdle PD. Systemic cytomegalovirus infection complicating ulcerative colitis: a case report and review of the literature. Postgraduate Medical Journal. 2004; 80 (942): 233-5.

5. Kambham N, Vij R, Cartwright CA, Longacre $\mathrm{T}$. Cytomegalovirus infection in steroid refractory ulcerative colitis: a case-control study. Am Jour of Surg Pathol 2004; vol. 28 (3): 365-73.

6. Kangro HO, Chong SKF, Hardiman A, et al. A pro- spective study of viral and mycoplasma infections in chronic inflammatory bowel disease Gastroenterology 1990; 98: 549-53.

7. Khan R, Rudkin P, Grewal K, et al. Cytomegalovirus colitis following azacitidine therapy. Can J Infect Dis Med Microbiol 2011; 22 (3)

8. Kim YS, Kim YH, Kim JS, et al. Cytomegalovirus infection in patients with new onset ulcerative colitis: a prospective study.; IBD Study Group of the Korean Association for the Study of Intestinal Diseases (KASID), Korea. Hepatogastroenterology. 2012 Jun; 59 (116): 1098-101.

9. Kuwabara A, Okamoto H, Suda T, Ajioka Y, Hatakeyama K. Clinicopathologic characteristics of clinically relevant cytomegalovirus infection in inflammatory bowel disease. Journal of Gastroenterology 2007; 42 (10): 823-9.

10. Lawlor G, Moss AC. Cytomegalovirus in Inflammatory Bowel Disease:Pathogen or Innocent Bystander? Inflamm Bowel Dis. September 2010; V.16 (9).

11. Mee AS, Jewell DP. Factors inducing relapses in IBD. Br Med J 1978; 2: 6140: 801-2.

12. Nguyen M, Bradford K, Zhang X, Shih DQ. Cytomegalovirus Reactivation in Ulcerative Colitis Patients Ulcers. 2011 January 1; 2011. doi:10.1155/2011/282507.

13. Orvar K, Murray J, Carmen G, et al. Cytomegalovirus infection associated with onset of inflammatory bowel disease. Dig Dis Sci 1993; 38: 2307-10.

14. Powell RD, Warner NE, Levine RS, et al. Cytomegalic inclusion disease and ulcerative colitis. Report of a case in a young adult. Am J Med 1961; 30: 334-40.

15. Traylen CM, Patel HR, FondawW, et al. Virus reactivation: a panoramic view in human infections Future Virol. 2011 April; 6 (4): 451-63.

16. Yee YK, Wong SW, Szeto ML. Ulcerative colitis exacerbation associated with cytomegalovirus infection. HKMJ 1998; 4: 437-9. 